Les plaques par contact direct ou impression furent préparées avec le fromage à chaque examen, et étudiées; elles sont représentées sur les clichés.

Le fait le plus significatif de l'étude préliminaire est la constatation que toutes les espèces bactériennes isolées de ce fromage type de Cheshire, par les méthodes quantitatives sont des formes en bâtonnets.

(A suivre)

\title{
UNE APPLICATION DE LA CHIMIE COLLOIDALE: L'ULTRAFILTRATION
}

\author{
par M. GEORGES GÉNIN,
}

Ingénieur, Eøole de Physique et de Ch'mie Industrielles de Par's

Il y a une vingtaine d'annóes, le terme colloïde n'était pour ainsi dire pas connu et rares étaient les ouvrages ou les travaux scientifiques dans lesquels la chimie colloïdale était citée.

Depuis cette époque, les choses ont bien changé et peut-être même emploie-t-on le mot colloïde dans des cas où la chimie colloïdale n'a rien à voir. Quoiqu'il en soit, l'étude de cette nouvelle branche de la chimie a permis de faire dans de nombreux cas de très grand progrès dans l'explication de phénomènes restés obscurs et a permis également de mettre au point des méthodes ou des techniques nouvelles dont certaines mêmes ont reçu des applications industrielles.

Parmi ces dernières, l'ultrafiltration est une de celles qui malgré sa mise au point relativement récente s'est montré z excessivement fertile en applications tant au laboratoire que dans l'industrie.

Si le terme ultrafiltration, ou filtration sur membrane constituée par un gel colloïdal, ne date que de 1905, à la suite d'un mémoire publié par Bechhold, l'emploi des membranes ultrafiltrantes remonte à beaucoup plus tôt et c'est en effet en 1855 que FIOK (1), pour la première fois, utilisa une membrane artificielle pour remplacer les membranes dialysantes animales ou végétales qu'avait signalé GraHAm dans son fameux mémoire sur la dialyse. Les études de Fick furent poursuivies quelques années plus tard à l'étranger par ScHumacher (2) qui employait des membranes de nitrocellulose et par Baranetzki en 1872 (3). En France, ce fut Martin (4) qui le premier eut, en 1896, l'idée de réaliser une filtration sur masse colloïdale, perméable aux cristalloïdes et imperméable aux colloïdes, afin d'assurer la séparation de deux éléments : neurotoxine et hemorrhagine qui existent dans le venin de certains serpents d'Australie.

Toutefois, c'est MaLfitano (5) qui eut vraiment l'idée de l'importance que pouvait jouer l'ultrafiltration dans les recherches scientifiques et en particulier dans l'étude des collö̈des et c'est à lui que l'on doit la 
mise au point et une méthode relativement simple de fabrication des membranes ultrafiltrantes. Le gel colloïdal employé par MaLfitano était un gel de nitrocellulose, et à l'heure actuelle, on peut admettre que seuls les dérivés cellulosiques sont utilisés pour la fabrication des membranes ultrafiltrantes. Le mode opératoire de MaLfitano consistait à étendre sur l'extrémité d'un tube à essais une solution de nitrocellulose dans un mélange d'alcool-éther, à laisser sécher incomplètement cette solution, à la coaguler dans de l'eau, et à retourner ensuite la pellicule de nitrocellulose ainsi formée, de façon à avoir un doigt de gant qui, était ensuite fixé à l'extrémité d'un tube de verre.

Il est bien évident qu'une telle membrane ultrafiltrante ne pouvait supporter que des pressions de filtration très faibles et ne pouvait donner que des très potits débits utilisables seulement pour des recherches de laboratoire.

Les études de Malfitano sur les applications des ultrafiltres à la chimie des colloïdes avaient déjà donné des résultats intéressants quand parut en 1906 un mémoire de H. BECHHOLD contenant la description et les applications d'un appareil beaucoup plus compliqué que celui de MaLfitano et dont l'usage ne s'est pas répandu dans les laboratoires (6).

Depuis cette époque, le nombre des dosuments relatifs à la fabrication des membranes ultrafiltrantes s'est multiplié et parmi les es sais les plus intéressants, il faut citer ceux de Grenet (7) et Selimbent dans lesquels la membrane est préparée en imprégnant de collodion soit une bougie de porcelaine soit un papier filtre, soit une capsule de Soxhlet et les travaux d'Ostwald qui, dans son Traité de manipulations de chimie colloïdale, a donné un assez grand nombre de méthodes pour la préparation des membranes de collodion.

Un grand progrès dans les possibilités d'emploi de l'ultrafiltration fut réalisé lorsque l'on put fabriquer d'une façon industrielle des membranes pouvant être employées d'abord en laboratoire, puis plus tard dans l'industrie. La fabrication d'une membrane de collodion est en effet pour une personne peu habituée à cette manipulation une opération assez délicate. De plus, malgré les règles qui ont été indiquées par le Professeur Asheshoy pour se mettre à l'abri des variations des conditions extérieures, il est très difficile, pour ne pas dire impossible, de préparer à plusieurs jours d'intervalle des membranes possédant des propriétés identiques. Cet inconvénient est très grave, en particulier dans certains cas dans lesquels il est de toute nécessité de pouvoir faire des mesures comparables.

A l'heure actuelle, la fabrication industrielle des membranes est résolue et diverses Maisons françaises et étrangères fournissent aux laboratoires comme à l'industrie des membranes de porosité variable et de propriétés bien définies. 


\section{Fabrication des membranes ultrafiltrantes}

Ainsi que nous l'avons dit plus haut, la plupart des membranes ultrafiltrantes sont préparées en partant de collodion cellulosique et plus particulièrement de collodion nitro-eellulosique. Un tel collodion est, comme on le sait, obtenu par la dissolution colloïdale de nitrocellulose dans un mélange alcool-éther. Ce collodion se présente sous la forme d'un liquide visqueux qui, versé en couche mince sur une surface plane et évaporé à une température ordinaire, laisse une pellicule de nitrocellulose assez résistante qui a trouvé aujourd'hui un emploi très important dans l'industrie des vernis.

La résistance mécanique de ce film est d'autant plus grande que le coton initial contient moins d'alcool. Ceci s'explique en effet facilement car l'expériense montre que le séchage de la pellicule s'effectue en deux temps: l'éther beaucoup plus volatil s'évapore d'abord, tandis que l'alcool est retenu en raison d'une part de sa tension de vapeur inférieure à celle de l'éther et d'autre part en raison du froid produit par l'évaporation de cet éther. Il en résulte que la nitrocellulose insoluble dans l'alcool se solidifie en donnant un gel et la concentration de ce gel, c'est-à-dire sa résistance mésanique, est d'autant plus grande qu'il y a peu d'alcool dans le mélange initial.

Si au lieu de laisser l'alcool s'évaporer complètement, ce qui donne une pellicule imperméable, on arrête l'évaporation après le départ de l'éther en coagulant le collodion au moyen d'eau par exemple, on obtient des membranes qui ont la structure d'une éponge extrêmement fine et qui sont percées d'un très grand nombre de canaux excessivement fins dont le diamètre est de l'ordre de quelques millionièmes de millimètre.

La composition initiale du collodion n'intervient pas seulement sur la résistance mécanique des membranes, mais intervient également sur la dimension des pores de la membrane. D’une façon générale, celle-ci a des pores d'autant plus gros que la proportion d'alcool est plus élevée et celle de la nitrocellulose faible. Inversement, lorsque le collodion contient un grand pourzentage d'éther, sa résistance mésanique est élevée, mais sa porosité est faible.

Il existe d'autres méthodes qui permettent de faire varier la porosité. L'expérience a en effet prouvé que plus le degré d'humidité de la pièce dans laquelle se fait la fabrication des membranes est faible, plus la perméabilité de la membrane est petite ; au contraire, plus cette humidité est importante, plus la porosité de la membrane est élevée. On peut également faire varier cette porosité en ajoutant au collodion lui-même différents liquides, comme l'eau, par exemple, qui augmentent très notablement le degré de perméabilité de la membrane.

Parmi les travaux les plus importants qui ont été publiés concernant les méthodes aptes à faire varier la porosité des membranes, il faut citer 
ceux de Bigelow et Gemberling, dans lesquels on fait varier la nature de la nitrozellulose (8), ceux de Malfitano (9), Eggerth (10), Nelson Morgan (1I) dans lesquels le même résultat est atteint en faisant varier la nature du solvant et la composition de ce dernier, ceux de Walpole (12), de Brown (13) et de BECHHOLd (14) dans lesquels pour faire varier les dimensions des pores, on agit sur la durée de séchage du collodion ou sur la conzentration de la nitrosellulose, ceux enfin de Schoep (15) et de Asheshov (16) dans lesquels l'addition de diluants tels que le glycérol ou l'eau au collodion augmente la porosité des membranes au détriment de leur résistance.

A l'heure astuelle, et malgré le grand nombre de brevets pris sur cette question, il n'y a guère que deux méthodes qui sont utilisées pour la fabrication des membranes industrielles, ce sont celle du Professeur Zsigmondy (17) dans laquelle chaque membrane est faite séparément en évaporant dans des moules circulaires un film de nitrocellulose et en le congelant dans de l'eau, et celle du Professeur Duchaux (18) qui présente l'avantage très considérable d'être continue et de permettre la préparation, en une seule opération, de plu-

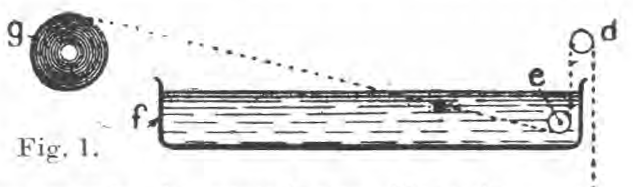
sieurs dizaines de mètres carrés de membrane filtrante dont toutes les parties sont absolument identiques. L'appareil du Professeur Duclaux se compose (Fig. 1) d'un cylindre $a$ sur lequel est enroulé le tissu servant de support. Ce tissu plonge dans un bae à collodion $b$, s'imprègne de collodion et passe ensuite par l'intermédiaire de rou-

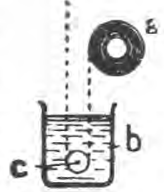
leaux de renvoi dans un bain de coagulation $f$ contenant de l'eau. La membrane après coagulation et lavage s'enroule, terminée, sur un rouleau magasin $g$.

Un certain nombre de recherches ont été faites au cours de ces dernières années pour essayer de remplacer la nitrocellulose qui possède certains insonvénients dans la fabrication des membranes ultrafiltrantes. Les seuls rézultats qui ont été publiés sont relatifs à la fabrication des membranes de cellulose qui d'ailleurs sont préparées en partant des membranes de nitro zellulose et en dénitrant ces dernières; à la fabrication de membranes à base de latex de caoutchouc (19) qui sont actuellement employées pour séparer les différents compartiments des bacs d'aceumulateurs et à la fabrication de membranes en nickel catalytique (20) qui sont préparées en déposant sur une toile métallique de nickel par un prosédé électrolytique du nickel pulvérulent qui, suivant lintensité du courant et la composition du bain électrolytique, possède des propriétés ultrafiltrantes très intéressantes.

On s'est demandé à plusieurs reprises quelle était la structure intime 
des membranes et on a cherché également à mesurer le nombre et la dimension des pores de ces membranes. Les recherches sur ce point ne sont pas encore terminées; toutefois il semble que les travaux les plus rézents aient confirmé que les membranes se présentent comme formées par une pellicule très peu épaisse traversée par une infinité de petits canaux rectilignes et fonctionnant tous comme des canaux capillaires (21). Lorsque l'on étudie en effet les variations de débit des membranes en fonction de la température, de la pression et de la viscosité des liquides, on constate que les résultats obtenus suivent très exactement la loi de Poiseuille. On peut donc en déduire que les canaux des membranes agissent comme des tubes capillaires et sont en particulier rectilignes, dirigés perpendiculairement à la plus grande face des membranes et non pas placés comme le sont les canaux des filtres en papier par exemple.

En ce qui concerne le nombre et le diamètre de ces canaux, les recherches récentes de İтсноок (22) ont montré que les pores des membranes de collodion ont un diamètre qui varie suivant leur préparation entre 1 et 200 millionièmes de millimètre et que leur nombre varie par $\mathrm{cm}^{2}$ entre $7 \times 10^{10}$ et $270 \times 10^{10}$.

\section{Les ultrafiltres et leur emploi dans les recherches de laboratoire}

Le nombre des appareils de laboratoire utilisant les membranes ultrafiltrantes de collodion s'est considérablement accru ces dernières années. D'ailleurs, tous les filtres à membrane de collodion de laboratoire se ramènent à trois types généraux qui sont : le petit filtre-presse dans lequel on remplace les toiles filtrantes ordinaires par une membrane, les ajpareils ultrafiltres à forme de bougie dans lesquels la paroi poreuse de porc-laine est remplacée par une masse de collodion reposant sur une armature et enfin les entonnoirs à filtration dans le vide dans lesquels par un disposit:x spécial, on monte des plaques ultrafiltrantes, à la place du papier filtre ordinairement employé.

Les appareils du premier type dont la figure 2 représente un spécimen sont généralement utilisés pour la mise au point en laboratoire des problèmes d@ filtration industrielle. Ces appareils en effet sont absolument conçus comme le sont les appareils d'un plus fort débit et les résultats qu'ils donnent permettent d'obtenir par un simple calcul ceux que l'on pourrait obtenir avec un filtre d'une surface filtrante supérieure. Ces appareils également sont parfois employés dans la petite industrie pharmaceutique ou en parfumerie, lorsqu'il faut filtrer quelques litres ou même quelques dizaines de litres par jour; ils peuvent en effet fonetionner sous une pression de plusieurs centaines de grammes par centimètre carré et ils sont munis en outre d'un certain nombre de robinets qui permettent de s'en servir, soit comme des filtres ordinaires, soit au contraire comme des dialyseurs, en remplaçant les membranes de collodion par des membranes dialysantes plus fines. 
Les bougies ultrafiltrantes ont le gros avantage de supporter une membrane très résistante, parce que imprégnant une âme constituée par des fils de soie tressés. Un de ces appareils dont la coupe est donnée sur la figure 3 est particulièrement employé pour la stérilisation des eaux d'alimentation et il donne en effet, avec un débit sensiblement identique à celui des bougies Chamberland une eau parfaitement stérile. De plus, l'efficacité de ces bougies est indéfinie, contrairement à ce qui se passe pour les bougies de porcelaine qui, au bout d'un certain temps, sont traversées par les microbes; pour les nettoyer, il suffit de passer à la partie extérieure de la bougie une éponge imbibée d'eau chaude, afin d'éliminer les sédiments retenus.

Les appareils basés sur le fonctionnement des entonnoirs à vide sont les plus nombreux et cette variété a permis d'en tirer le meilleur parti pour la recherche scientifique et pour l'analyse. Ces appareils,

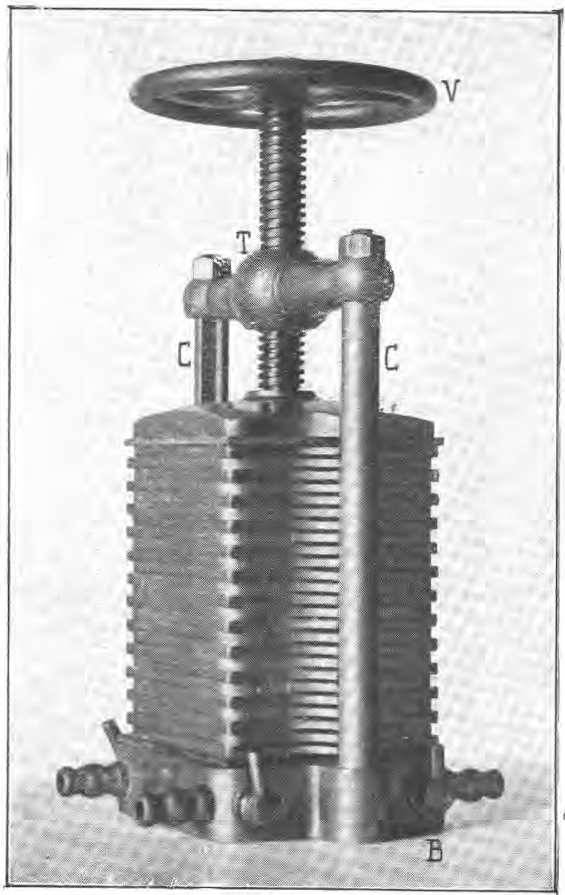

Fig. 2. en effet, suivant les modèles, ont une surface filtrante qui varie entre 1 et quelques dizaines de $\mathrm{cm}^{2}$ et peuvent supporter, suivant les cas, des pressions de quelques centaines de grammes, jusqu'à plusieurs

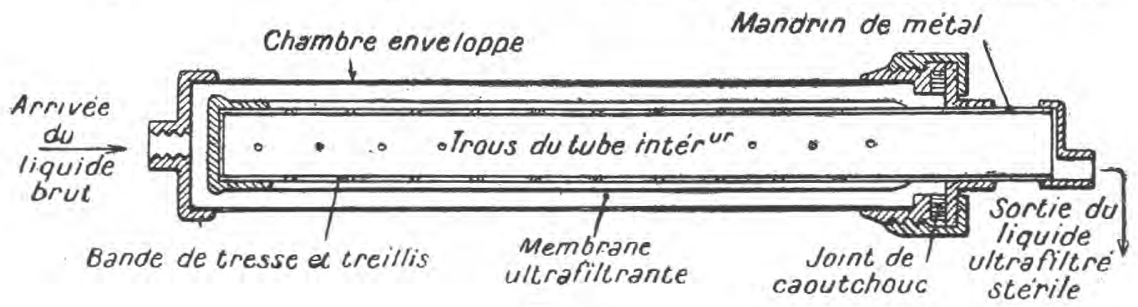

Fig, 3.

centaines d'atmosphère par $\mathrm{cm}^{2}$. On peut donc monter sur ces appareils des membranes très serrées et utiliser de très fortes pressions de filtration, ou au contraire des membranes très lâches fonctionnant presque par 
simple gravité. On peut en outre traiter soit plusieurs centaines de $\mathrm{cm}^{3}$ et eoncentrer ainsi sur un très petit espace la totalité des sédiments contenus dans un liquide, ou au contraire, nopérer que sur quelques centimètres cubes et appliquer dans certains cas l'ultrafiltration aux méthodes de la micro-analyse.

Les appareils les plus répandus sont les appareils Thiessen (fig. 4), les appareils du Professeur Sterner (fig. 5), l'appareil Buwa, l'ultrafiltre de l'Institut Agrieole de Braunschweig, les appareils du Professeur Zsigmondy, et l'appareil de Brukner et Overbeck.

L'appareil Thiessen est plus spécialement indiqué pour la micro-

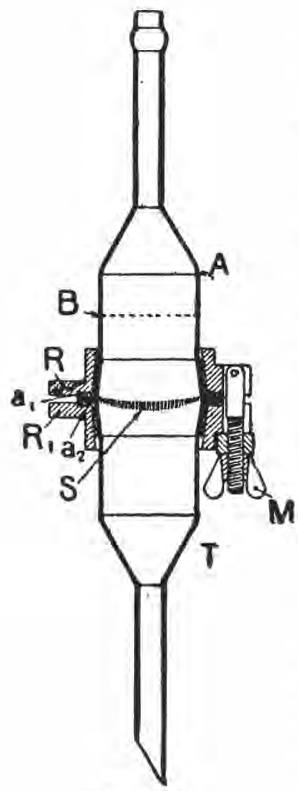

Fig. 4. analyse et les recherches bactériologiques. Il possède en effet le gros avantage de pouvoir être stérilisé par la vapeur, il se compose d'un entonnoir $\mathrm{T}$ et d'un réservoir cylindrique destiné à contenir le liquide à filtrer. Entre l'entonnoir et le réservoir, on place la membrane de collodion possédant une surface filtrante d'environ $3 \mathrm{~cm}^{2}$ et soutenue par un disque perforé concave qui permet de recueillir le précipité au centre même de la membrane, et d'éviter sa répartition sur toute la surface. L'appareil se monte sur un vase à filtration si l'on désire filtrer par aspiration, ou au contraire, il peut être relié à une source de gaz comprimé, si l'on désire filtrer sous pression.

L'appareil de SteIner est une modification de l'appareil Thiessen et permet, en réduisant à leur strict minimum les manipulations indispensables, de filtrer des liquides contenant des bactéries. Il se compose du réservoir proprement dit de l'appareil Thiessen surmonté d'une cloche spéciale portant un ajutage dans lequel s'engage l'extrémité d'un résipient destiné à faire des ponctions. Un tel dispositif permet le passage direct dans l'ultrafiltre des liquides extraits par ponction sans aucune altération de ces liquides.

L'appareil Buwa (fig. 6) est également employé d'une façon courante dans l'analyse quantitative, mais à l'encontre des deux appareils décrits précédemment, il n'est pas stérilisable par la vapeur, par suite des pièces de caoutchouc qu'il contient. Il se compose d'un tube très fin surmonté d'un renflement portant à la partie supérieure un disque de porcelaine perforé. Ce tube est placé à force dans un bouchon de caoutchouc monté lui-même à l'intérieur d'un tube en verre de grand diamètre. Sur le disque perforé, on place une petite feuille de papier filtre, puis une membrane de collodion et on applique le tout sur le bouchon de caout- 
chouc au moyen d'un cylindre en verre maintenu au contact du bouchon par l'intermédiaire de deux ressorts.

L'appareil qui a été construit par l'Institut Agricole de Braunschweig est destiné à la filtration des bactéries excessivement petites, aussi comme on est obligé d'employer des membranes de très faible perméabilité, il faut pour obtenir des débits intéressants filtrer sous très forte pression et l'appareil est prévu pour fonctionner sous une pression de 5 atmosphères. Ses prinsipales applications sont les recherches microbiologiques et sérologiques, et il a été construit spécialement pour permettre la recherche rapide des bacilles de la tuberculose dans les expectorations et le lait des bovidés. Il est entièrement métallique et peut être stérilisé à la vapeur.

Les appareils du Professeur ZsIGMONDY ne sont pas autre chose que des entonnoirs à filtration dans le vide décomposés en 3 parties: l'entonnoir proprement dit, le disque de porcelaine perforé et le cylindre dans lequel on place le liquide à filtrer. Il est nécessaire d'utiliser des appareils de cette forme, car si l'on place au fond d'un Buchner une membrane de collodion sans aucune présaution spésiale, la membrane ne colle pas sur le disque perforé et il se produit des fuites. Il faut absolument, ainsi qu'il est montré sur la figure avoir un appareil démontable et permettant de faire un joint parfait lorsqu'on applique, au moyen d'un double collier de serrage, le cylindre sur la plaque perforée. Ces appareils sont construits le plus souvent en por-

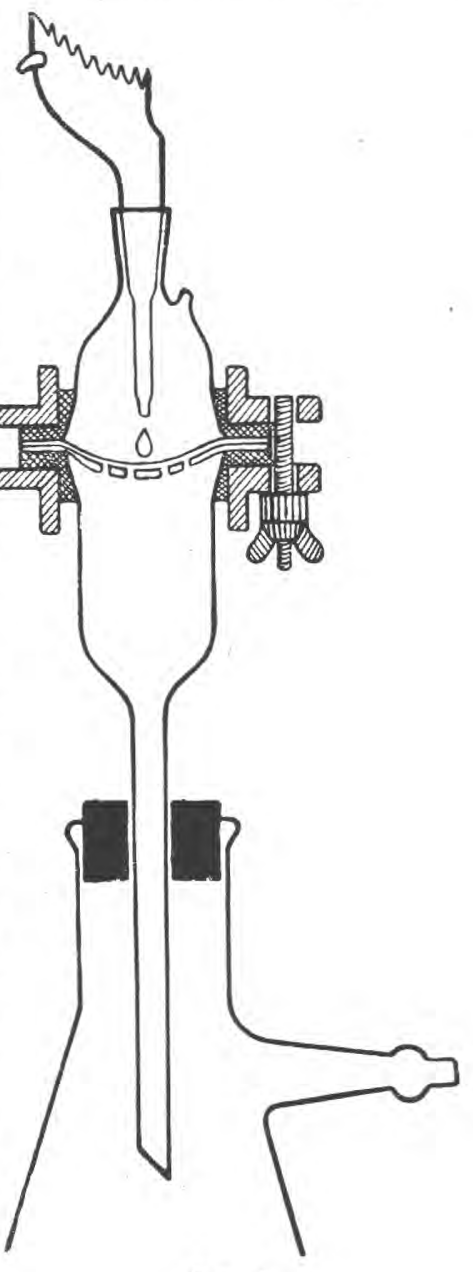

Fig. 5. celaine, mais on en rencontre également en ébonite ou en bronze nickelé. Leur principal emploi réside dans l'analyse qualitative et dans la recherche scientifique (fig. 7).

A côté de ces appareils, que l'on rencontre le plus souvent dans la littérature chimique, il en existe beaucoup d'autres, tels que l'ultrafiltre de Bruckner et Overbeck, l'ultrafiltre de BechHold, de Asheshov, ete., 
qui ont été construits pour des buts bien déterminés et pour des problèmes particuliers d'analyse chimique ou de bactériologie.

\section{Ultrafiltres industriels}

Les ultrafiltres de Iaboratoire que nous avons décrits plus haut donnent des débits évidemment très faibles et même multipliés, ils ne

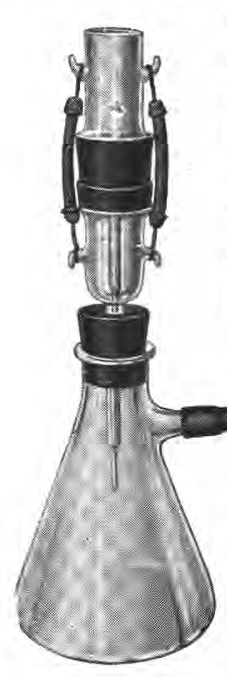

Fig. 6. pourraient donner les débits élevés qui sont exigés par l'industrie chimique d'aujourd'hui. Aussi devant le développement de la technique de l'ultrafiltration en laboratoire, on a cherché à appliquer cette technique aux problèmes industriels, par l'utilisation de membranes de grande surface filtrante et d'une résistance méca. nique suffisante pour pouvoir résister à des manipulations journalières pendant un temps suffisamment long.

Les essais qui ont été faits dans cette voie sont assez nombreux et on a cherché bien entendu à monter des membranes ultrafiltrantes sur les appareils les plus divers d'où le nombre assez élevé de brevets pris sur cette question.

Il semble aujourd'hui que le problème ait été résolu par l'emploi de filtres presses ayant subi quelques modifications et dans lesquels, on remplace les toiles filtrantes ou les papiers filtres par des membranes de collodion.

Ces ultrafiltres presses ne diffèrent en réalité que par quelques points de détails des filtres presses courants; ils sont en général mieux construits que les filtres presses et en particulier beaucoup mieux dressés, car dans ces appareils, le joint entre les plateaux consécutifs n'est fait que par une membrane de collodion, c'est-à-dire par un joint d'une épaisseur de quelques dixièmes de millimètre. On a prévu en outre des dispositifs qui permettent de faire la purge de l'air contenu dans l'intérieur de l'appareil, car les membranes de collodion sont imperméables à l'air et si celui-ci ne pouvait s'échapper, il y aurait formation à l'intérieur du filtre d'une poche d'air comprimé qui s'opposerait à la filtration. Enfin, suivant les cas on prévoit quelques dispositifs spéciaux, en particulier lorsqu'il faut retirer le gâteau formé à l'intérieur des cadres par démontage du filtre sans abîmer la membrane par des démontages successifs. 
En ce qui concerne les dimensions de ces appareils, on rencontre actuellement des ultrafiltres-presses dont les plateaux ont depuis $0 \mathrm{~m}$., 30 de côté jusqu'à 1 mètre de côté. Les bâtis de ces filtres pouvant contenir jusqu'à 80 éléments de filtration, il en résulte que la surface filtrante de ces appareils varie depuis $1 \mathrm{~m}^{2}$ pour les ultrafiltres du plus petit modèle jusqu'à $30 \mathrm{~m}^{2}$ et même plus pour les appareils plus considérables (fig. 8).

Enfin, ces appareils sont construits usuellement en bois, en fonte, en aluminium, en bronze, etc., mais rien n'empêche, s'il le fallait, de les construire enunautre métal ou en une

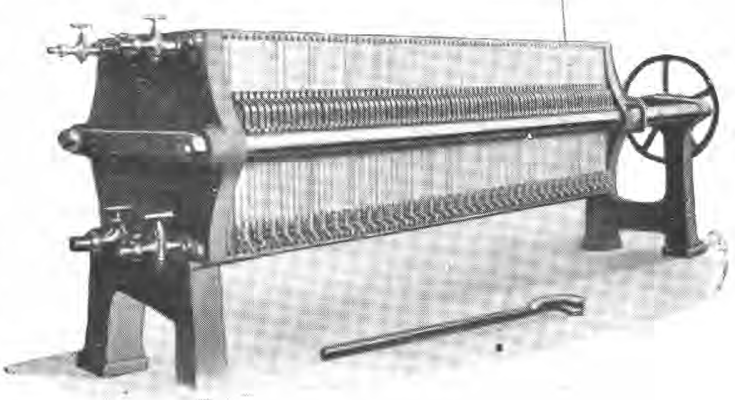

Fig. 8. substance plastique, genre ébonite, ou bakélite. Nous verrons plus loin quels sont les débits que peuvent donner ces appareils et leurs principales applications dans I'industrie chimique.

\section{Emploi de l'ultrafiltration dans les recherches de laboratoire}

Nous avons vu plus haut que les ultrafiltres de laboratoire dont la description a été donnée, sont utilisés soit pour la mise au point en laboratoire de problèmes de filtration industrielle, soit pour la stérilisation des eaux d'alimentation, soit enfin pour les recherches scientifiques.

Nous donnerons quelques indications complémentaires concernant ces applications plus spéciales des membranes ultrafiltrantes espérant que nos lecteurs trouveront dans la liste déjà longue des travaux publiés sur cette question, des indications précieuses pour leurs recherches personnelles.

Deux domaines de la recherche scientifique utilisent l'ultrafiltration, ce sont, d'une part, l'analyse chimique et la microanalyse, qui profitent de la propriété des membranes d'arrêter les colloïdes, et par conséquent les précipités colloïdaux, et d'autre part, la chimie biologique qui trouve dans l'ultrafiltration un moyen de concentrer, sous un faible volume et par conséquent d'étudier avec plus de facilité, les substances colloïdales que l'on trouve en très grand nombre dans la matière vivante.

Parmi les nombreux travaux que nous signalerons en ce qui concerne l'analyse, il faut citer ceux de VIKTor et Ivesco (23), sur la filtration des colloïdes par les membranes, de FABRE et RENAU (24) sur un procédé de dialyse rapide et son application à la préparation $\mathrm{du} \mathrm{Fe}^{2} \mathrm{O}^{3}$, ceux de FOUARD (25) relatifs à l'analyse physico-chimique des solutions, ceux 
de HABER (26) qui contiennent des données particulièrement intéressantes concernant l'analyse des dispersoïdes, ceux de BachmanN (27), de Hern et Spate (28) sur l'ultrafiltration des solutions collö̈dales, des dérivés organochromiens dans le bromure d'éthylène, ceux de HALM (29) sur l'analyse technique des dispersoïdes, ceux de ZaKarias (30), ceux de JANDER (31) sur l'emploi des membranes ultrafiltrantes dans l'analyse quantitative, ceux de VIEGNer (32) sur l'emploi des membranes de collodion dans la chimie analytique, ceux enfin de Wolski (33) de Stulhmann (34), de Hatschek (35), de Bachmann (36), de Mosev (37), tous relatifs à des applications de l'ultrafiltration à des problèmes de chimie colloïdale.

La bibliographie relative à l'emploi des membranes ultrafiltrantes dans les recherches de chimie biologique et de bactériologie est tout aussi nombreuse. Toutefois dans ce cas, les travaux publiés sur cette question se trouvent rassemblés dans des traités dont les principaux sont ceux de Rheinhold (38), méthodes de dialyse et d'ultrafiltration, d'Ostwald dans son Traité pratique de chimie colloïdale, de JANDER dans son Traité de méthodes de travail de la chimie générale, de Zsigmondy dans son Traité de chimie colloïdale, de Houben Weyc dans son livre intitulé "Les méthodes de la chimie organique ", de Bachmann dans son Traité des méthodes de recherches colloïdochimiques. A côté de ces traités généraux, il faut également citer quelques articles originaux relatifs à des points spéciaux, qui sont d'ailleurs le plus souvent signalés dans les Traités que nous venons d'énoncer.

On voit don s par cette courte énumération que malgré sa jeunesse, la technique de l'ultrafiltration a déjà donné naissance à de nombreux travaux et a pərmis de résoudre aisément dans bien des cas des problèmes que les méthodes générales de la chimie ne parmettaient de résoudre qu'avec beaucoup de difficultés.

\section{Les applications industrielles de l'ultrafiltration}

Actuellement, les applications industrielles de l'ultrafiltration ne sont pas très nombreuses. Cependant, des études sont en cours et font prévoir dans un avenir rapproché un grand développement de l'emploi des membranes de collodion dans l'industrie chimique et dans l'industrie alimentaire.

Parmi les industries qui utilisent couramment les membranes de collodion, il faut signaler l'industrie des huiles végétales comestibles qui trouve dans l'ultrafiltration un procédé parfait pour donner aux huiles comestibles une limpidité complète et surtout définitive. A côté de ces huiles végétales, il faut citer également l'application des membranes aux huiles minérales (huile de paraffine, huiles de graissage, huiles électriques), aux huiles pharmaceutiques (minérales, animales et végétales), et enfin l'application à la récupération des huiles usagées. 
A côté de l'industrie des huiles, il faut citer comme autres branches qui commencent à employer les membranes l'industrie de la parfumerie, pour la purification des eaux de Cologne, des lotions, des extraits, des huiles essentielles, l'industrie des produits pharmaceutiques en général et quelques industries de produits chimiques.

Enfin, on espère proshainement mettre au point dans des conditions industrielles la filtration de toutes les boissons fermentées ou non, et en particulier la filtration des bières, des vins, des moûts, et des jus de fruits. Cela est un très gros problème et s'il était résolu par l'affirmative, l'ultrafiltration amènerait dans toutes ces industries une amélioration considérable des méthodes de travail et une facilité d'opération actuellement très recherchée.

En ce qui concerne les débits de ces appareils, ils varient bien entendu avec les surfaces filtrantes, mais on peut citer comme appareils usuels des ultrafiltres-presses pour la filtration des huiles qui débitent 10 tonnes d'huile par 24 heures, soit huile minérale, soit huile végétale, des appareils qui dans l'industrie de la parfumerie débitent 100 à 200 litres à l'heure de lotion ou d'eau de Cologne, et enfin dans l'industrie des produits pharmaceutiques des filtres d'une surface filtrante beaucoup plus réduite dont le débit varie depuis quelques litres jusqu'à quelques centaines de litres à l'heure. On voit par ces chiffres que l'ultrafiltration a nettement quitté le domaine du laboratoire et qu'elle permet d'obtenir des résultats qu'il aurait été bien difficile de prévoir lorsque Malfitano commencait ses recherches sur cette question.

\section{L'ultrafiltration et I'industrie laitière}

Lə développement de l'ultrafiltration est encore trop récent pour que cette technique ait pris dans toutes les branches de l'industrie une place primordiale. En particulier dans l'industrie laitière, l'ultrafiltration n'a fait que de très brèves apparitions et surtout lorsqu'il s'agissait de recherches scientifiques. Nous avons cité plus haut quelques travaux faits au moyen des membranes et trouvant leur intérêt dans l'étude du lait et de ses dérivés.

En ce qui conserne l'industrie proprement dite, il apparaît difficile à première vue de signaler un rôle possible de l'ultrafiltration. Cependant, tout rézemment vient d'être publié un brevet anglais de concentration du lait par un procédé mixte d'évaporation et d'ultrafiltration. Dans ce brevet (39), l'ultrafiltration permet de séparer, d'un côté, les matières salines et les sucres en solution dans le lait et, de l'autre côté, les matières grasses : albumine, caséine, en suspension dans le lait.

Peut-être également, l'ultrafiltration sera employée pour la préparation et la stérilisation du sérum du lait, mais actuellement, cette application est encore dans le domaine du laboratoire. 
Nous avons voulu, dans ce qui précède, décrire assez complètement un procédé nouveau et signaler non pas ses applications actuelles, mais surtout attirer l'attention de nos lecteurs sur les possibilités d'emploi de ce procédé que nous estimons plein d'avenir.

\section{Bibliographie}

(1). Ann. Phys. u. Chem. 94, p. 59, 1855.

(2) Ann. Phys. u. Chem. 110, p. 337, 1860.

(3) Ann. Phys. u. Chem. 147, p. 195, 1872.

(4) J. Physiol. 20, p. 364, 1896.

(5) Rev. Gen. Sci. 99, p. 617, 1908.

(6) Z. Physik. Chem. 40, p. 257, 1907.

(7) Ann. Inst. Pasteur, p. 240, 1908.

(8) J. Am. Chem. Soc. 29, p. 1576, 1907.

(9) Loc. cit.

(10) J. Biol. Chem. 48, p. 203, 1921.

(11) J. Biol. Chem. 58, p. 305, 1923.

(12) Biochem. J. 9, p. 284, 1915.

(13) Biochem. J. 9, p. 591, 1915.

(14) Biochem. Z. 6, p. 379, 1908.

(15) Koll. Z. 8, p. 80, 1911.

(16) Compt. Rend. Soc. Biol. 92, p. 362, 1925.

(17) Br. Fr. 515.092, 17-12-17.

(18) $\mathrm{Br} . \mathrm{Fr} .567 .970$.

(19) $\mathrm{Br} . \mathrm{Fr}$ 605.245, 21 mai 1926.

(20) J. Chem. Soc., p. 1127, 1926.

(21) Rev. Gen. Coll., p. 130, 1924.

(22) J. Gen. Phys. 9, p. 755, 1926.

(23) Compt. Rend. Soc. Biol. 61, p. 197, 1906.

(24) Bul. Soc. Chim. Biol. 8, p. 201, 1926.

(25) J. Soc. Chem. Ind., p. 268, 1925.

(26) Koll. Z., 37, p. 377, 1925.

27) Chem. Zeit. 43, p. 595, 1919.

(28) Koll. Z. 28, p. 236, 1926.

(29) Koll. Zeit. 37, p. 377, 1925.

(30) Koll. Zeit. 37, p. 50, 1925.

(31) Z. für Anal. Chem. 61, p. 145, 1922.

(32) Koll. Z., p. 228, 1921.

(33) Mit. für Leben. und Hyg. 12, p. 263, 1921.

(34) Thèse Université de Göttingen, 1921.

(35) Z. für Chem. und Ind. der Koll. VI, No 5.

(36) Z. für Anorg. Chem. 103, p. 119, 1918.

(37) Chem. Zeit. 44, p. 637, 1920.

(38) Méthodes de la Chimie organique. I, p. 465, 1925.

(39) Br. Ang. 285.313. 